\title{
Los elementos filosóficos de El Estado comercial cerrado de Fichte
}

\author{
Philosophical elements of Fichte's The Closed Commercial State
}

\author{
Mariano Lucas Gaudio \\ mgaudio77@yahoo.com.ar \\ (Universidad de Buenos Aires/CONICET, Buenos Aires, Argentina)
}

\begin{abstract}
Resumen: En el presente trabajo se intenta mostrar el trasfondo filosófico del concepto de Estado racional en El Estado comercial cerrado (1800) de Fichte, en cuanto los problemas políticos y económicos se sustentan en la concepción metafísica de la Doctrina de la Ciencia. Y se analizan tres ejes: en primer lugar, lo ideal como fundamento de lo real, donde éste se explica desde un concepto de fin proyectado; luego, la doble contraposición frente al dogmatismo y la articulación entre lo universal y lo particular en el idealismo, que conduce a una visión del Estado como unidad fundante; y finalmente, el espacio comercial social - horizontal, donde la posición de cada particular está en relación recíproca con cada otro particular.
\end{abstract}

Palabras-clave: Fichte; Estado; metafísica; articulación; reciprocidad.
Abstract: This paper attempts to expound the philosophical background of the concept of rational State in Fichte's The Closed Commercial State (1800) and how the Science of Knowledge's metaphysics ground the political and economical problems found in that work. In order to achieve this, we analyze three points. In the first place, we study the conception of the ideal as the foundation of the real, which is explained through the concept of a projected final goal. In the second place, we compare dogmatism with the articulation between the universal and the particular in Idealism, which leads to a conception of State as a founding unity. In the third and last place, we analyze the commercial, social and horizontal space, where the position of each particular is in a reciprocal relationship with the others.

Keywords: Fichte; State; metaphysics; articulation; reciprocity.

En un momento bisagra de su producción filosófica, tras haber gozado de la repentina fama y del no menos repentino ocaso en Jena, J. G. Fichte publica El Estado comercial cerrado $(1800)^{1}$ como apéndice del Fundamento del derecho natural (1796-7), ${ }^{2}$ donde abre un plano específico, el de la política concebida como aplicación coherente de los principios jurídicos, y más concretamente, la realización de los mismos en la base económica. En este sentido, se puede interpretar El Estado

1 Der geschlossene Handelsstaat (1800). En Sämmtliche Werke (en adelante: FSW, tomo, página): FSW III 387-513. Las referencias completas de fuentes y bibliografía se hallan al final del artículo, en "referencias bibliográficas".

2 Grundlage des Naturrechts (1796-7) (en adelante: GNR). En Gesamtausgabe der Bayerischen Akademie der Wissenschaften (en adelante: GA, serie/tomo, página): GA I/3 313-469, I/4 5-165. 
comercial cerrado de Fichte como prolongación del Derecho natural o como una pieza independiente y autónoma. Pero en ambas perspectivas se pierde el anclaje propiamente especulativo, la sustancia metafísica de la obra fichteana. Y a partir de esta omisión, o de una mirada parcial sobre la filosofía de Fichte, surge un abanico de malentendidos sobre el concepto racional de Estado, en aspectos como el carácter autoritario o totalitario, compulsivo/coactivo, intervencionista, extremadamente planificador, etc. ${ }^{3}$

Un análisis de la obra en cuestión no puede no considerar los elementos filosóficos que están a la base de la exposición jurídica, política y económica, y lo que motiva el presente trabajo consiste precisamente en poner de manifiesto algunos vectores que amplían la concepción del Estado en Fichte en referencia a la Doctrina de la Ciencia, concibiendo a ésta no como una obra en particular sino como un sistema que se vale de una terminología fluyente. Tales elementos filosóficos se podrían esquematizar en torno de tres ejes: la relevancia del proyecto en cuanto idealidad fundante de realidad, la doble contraposición ante el dogmatismo a partir de la cual se comprende la articulación entre lo universal y lo particular, y la aplicación de esa misma articulación en un ámbito común-relacional. La reconstrucción siguiente trata de explicitar estos ejes fundamentales para una interpretación filosófica de El Estado comercial cerrado de Fichte.

I.

La relevancia de la idealidad se desprende del mismo subtítulo, puesto que El Estado comercial cerrado se define como un “proyecto filosófico”, una política a

3B. Willms (1967, pp.12-14, 80-90) reconstruye las tempranas interpretaciones sobre la filosofía política de Fichte y rebate la equiparación con el totalitarismo como variante del despotismo, basándose en el concepto de libertad, inescindible de la limitación y de la coacción. La argumentación de Willms resulta central para evitar el abanico de malentendidos que señalamos. Pese a ello, R. Gray (2003, p.538) equipara el Estado fichteano con la economía planificada del socialismo del siglo XX, B. Frischmann (2006, pp.580-581) deslinda tajantemente el modelo trascendental de su aplicación política como si ésta fuera inconsecuente con aquél, S. Gross (2008, pp.206-209) sostiene que el centralismo y el control colisionan con la libertad personal y la libertad de mercado, y D. Moggach (2009, p.1003), que en principio iguala el modelo de Estado con el intervencionismo despótico y después (p.1016) los distingue, al final (p.1019) critica la vigilancia y la centralización. Así, la observación de Willms contrasta con estas caracterizaciones negativas del Estado fichteano donde la referencia a la Doctrina de la Ciencia es nula o escasa. Lo último vale también para R. Gray, que curiosamente reprocha a A. Verzar (1980) no haber profundizado esta relación, y cuya propuesta consiste en igualar el primer principio, "Yo = Yo", con la fórmula "dinero = dinero" (pp.545-546), lo que es absurdo, porque en El Estado comercial cerrado el dinero se subordina a la política comercial estatal. En efecto, I. Nakhimovsky (2011, pp.355, 362 ss., 381-382, 407 ss.) subraya la relevancia del dinero pero como política de Estado, y en conexión con la Tathandlung de la Doctrina de la Ciencia propone el poder ejecutivo (pp.157-158). Además de afirmar que el Estado no interfiere en las libertades individuales sino que delimita y evita las obstaculizaciones recíprocas, concluye que esta obra no se asimila ni al socialismo ni al totalitarismo, sino que expresa una crítica al orden político-económico existente (pp.483, 510-512). 
realizar en el futuro, ${ }^{4}$ por parte de un Estado que ha alcanzado la unidad jurídica - la legislación común de una multiplicidad de hombres bajo un mismo poder coactivo - sin lograr aún la unidad económica fundamental y, por tanto, se ha unificado internamente sólo a medias. En la “Dedicatoria”, Fichte distingue y contrapone al filósofo especulativo y al político en ejercicio, que suelen relacionarse mediante ideas que el primero expresa y el segundo desecha por incumplibles, generando así un abismo infranqueable entre lo ideal y lo real. Sin embargo, si el filósofo no considera su saber como un mero juego, sino como algo serio, entonces jamás descarta su realizabilidad, y a lo sumo tendrá que afinar y precisar la universalidad abstracta con aspectos determinados, hasta que las ideas sean aplicables a la situación dada. Por contrapartida, el ámbito propio del político en ejercicio contiene un nivel de complejidad y de riqueza conceptual que se sustrae de lo general, porque - con la analogía de Fichte - conocer las relaciones y lados de un triángulo no significa conocer el valor de cada uno de ellos en particular, y del mismo modo tampoco sirve a tal fin apelar a un instrumento de medida, a una técnica pergeñada de antemano e independiente de la situación concreta (FSW III 389-391).

Ambos polos, la especulación y el ejercicio, componen el plano de la política y se necesitan mutuamente: sin la filosofía, la política se convierte en un saber particularista, de recetas quizás válidas en cierto tiempo pero incapaces de trascender la perspectiva meramente administrativa y, por lo tanto, mecanicista-dogmática; y sin la aplicación, la filosofía se convierte en una simple confesión de deseos, un saber estéril e incapaz de mostrar el pasaje de la determinabilidad a la determinación. Esta complementariedad mutua refleja tanto la necesaria dimensión filosófica de la política, como la importancia de llevar a la práctica las ideas. La tarea común consiste en articular lo universal con lo particular.

El carácter proyectivo de El Estado comercial cerrado remite a una serie de consideraciones de la Doctrina de la Ciencia nova methodo (1796 ss.). En efecto, en esta obra Fichte afirma que el Yo, que se pone, también se opone y coloca en la conciencia, en un mismo acto, los opuestos - el ponerse y la contraposición con lo múltiple - , como dos caras de un mismo proceso, de la actividad que también es reposo. Así, lo encontrado (lo dado, lo empírico) se erige a partir de lo proyectado, lo que se produce ante la mirada. Lo encontrado y lo proyectado se vinculan mediante la actividad que, en cuanto facultad práctica, se desenvuelve en sentido inverso al pasaje de lo determinable a lo determinado. ${ }^{5}$ En otras palabras, "la libertad es el punto de partida de toda filosofía” (WLnm-H § 2, GA IV/2 45). ${ }^{6}$ Pero la libertad, si

\footnotetext{
4 Así dice el subtítulo: "Un proyecto filosófico como apéndice a la Doctrina del Derecho y una prueba de la política a seguir en el futuro", FSW III 387.

5 Wissenschaftslehre nova methodo - Halle (en adelante: WLnm-H) § 2, GA IV/2 42-43.

6 Lo mismo en WLnm-H § 3, GA IV/2 47.
} 
no ha de ser meramente abstracta, tiene que articularse con la limitación, y con ésta surge lo encontrado. Ahora bien, el aparecer de lo encontrado presupone una actividad proyectada, o lo que es lo mismo, un concepto de fin. La práctica involucra a la teoría - la facultad de los conceptos o inteligencia - como un momento de su proceso de realización, que consiste en determinar lo determinable (WLnm-H § 4, GA IV/2 47-48, 50, 55).

Desde luego, la primacía práctica no significa que lo encontrado sea un invento del Yo, pues el Yo lo encuentra-lo siente, lo representa - de una determinada manera. Pero lo encuentra porque actúa, y si no actuara no lo encontraría. En este sentido, aunque el Yo se sienta coaccionado a representar el objeto tal como lo encuentra, en rigor sólo está en cierta medida condicionado, porque bien podría pensarlo con otras características, y entonces depende de la libertad si el Yo se apega y es fiel a la limitación tal como la encuentra o si decide modificarla idealmente en la conciencia, quizás para proyectar un concepto de fin que conlleve la transformación de lo dado en la realidad. ${ }^{7}$ Incluso en el nivel teórico más elemental interviene la libertad. Lo dado como tal responde a una proyección del Yo articulada con las determinaciones. Si el Yo no actúa, lo dado no aparece, y cuando lo dado aparece el Yo - por así decir - deja de actuar (o inhibe parcialmente su actividad, suspende la facultad transformativa y la proyección de un concepto de fin) para percibirlo y conocerlo, para configurar la determinada manera en que se presenta el objeto.

Al igual que la filosofía, la tarea de la política se perfila en la articulación entre lo universal y lo particular, y entonces la proyección ideal no tiene otro cauce que el de evidenciar el pasaje de la determinabilidad a la determinación, es decir, está motivada por la producción de acciones que transformen lo encontrado en función de una Idea. En consonancia, dice Fichte en El Estado comercial cerrado:

$\mathrm{Si}$, con el conocimiento correcto de la situación presente, fuera elaborada una política según esta Idea, por lo demás fundada desde firmes principios jurídico-estatales, y con las consecuencias de los mismos, entonces esta política podría parecer inútil sólo al mero empirista (FSW III 391).

El referente de "esta Idea" resulta ambiguo: a primera vista, aludiría a la conjugación entre regla y caso; pero también podría significar la propia concepción política de Fichte, que incluye lo especulativo y que desemboca en el cierre y autonomización comercial del Estado. De todos modos, refleja el carácter proyectivo y el contraste con el mero empirista sujeto a la repetición mecánica y tecnocrática de fórmulas abstractas preestablecidas. El mero empirista impugna todo concepto o cálculo que vaya más allá de los hechos, es decir, anula la dimensión productiva y creativa de realidad que distingue a la política. Además de copiar y mezclar la

7 WLnm-H § 7, GA IV/2 89. Sobre la necesaria referencia del sentimiento al Yo: § 6, GA IV/2 73-74; § 7, GA IV/2 86. 
receta con las condiciones sin comprenderlas ni interpretarlas, el empirista deviene administrador de lo dado, rechazando lo posible (el concepto de fin) como si fuera imposible. Incluso el reservorio de conocimientos al que apela desconoce su génesis histórica, el contexto en que una propuesta fue innovadora y que en la actualidad traería más problemas que soluciones. En última instancia, la posición del empirista se asemeja a la del dogmático, no sólo en cuanto alude al carácter predeterminado e inmodificable de lo dado, sino también en cuanto anula cualquier proyección y realización de la libertad en articulación con la experiencia. ${ }^{8}$

La argumentación filosófica de la "Dedicatoria" se completa con una argumentación nítidamente política. En la medida en que el proyecto de El Estado comercial cerrado consiste principalmente en delimitar las fronteras económicas e instaurar una moneda nacional, a semejante idea no se le podrá objetar que sea irrealizable, sino a lo sumo que atenta contra ciertos intereses. Y Fichte decide mostrar esos intereses: en verdad, la resistencia proviene de la gran ventaja que obtiene Europa sobre el resto del mundo, llevándose a muy bajo precio las fuerzas y los productos de las regiones sometidas, lo que atenta contra el derecho y constituye una contradicción imposible de perdurar (FSW III 392-393). Aunque aquí evite explicar la imposible continuidad de las colonias y del comercio de esclavos, los motivos se hallan en los escritos de revolución, ${ }^{9}$ y aunque aquí Fichte descarte una respuesta a la observación de sacarle provecho a esta ventaja mientras dure, en el desarrollo de El Estado comercial cerrado hay argumentos inconciliables con la expansión dominadora. ${ }^{10}$ Sea como fuere, no cabe desechar este proyecto filosófico por irrealizable, sino sólo por administrativismo y temor a las transformaciones sustanciales.

Si lo empírico y existente no se erige independientemente de la actividad que lo legitima, sino como aquello que encuentra el Yo porque proyecta un concepto de fin y asume el pasaje de la determinabilidad a la determinación, entonces lo real no se presenta como un dato último y definitivo, sino como resultado de una actividad que lo constituye. En esta perspectiva se comprende la afirmación de la “Introducción”: “El Estado real se puede representar, por tanto, como concebido en la instauración gradual del Estado racional” (FSW III 397). La política, que no se

8 Por eso dice Fichte: "Su enfermedad incurable es la de tener por necesario lo [que en verdad es] contingente" (FSW III 449).

9 Nos referimos a Zurückforderung der Denkfreiheit (1793): FSW VI 5, y Beitrag zur Berichtigung der Urteile des Publicums über die französische Revolution (1793): FSW VI 80-81, donde la opresión autoriza a la revolución y auto-afirmación de la libertad inalienable. La perspectiva se puede completar con Ueber Geist und Buchstab in der Philosophie (1795): FSW VIII 286-289, como desarrollo gradual de la libertad.

10 Para Fichte la apertura comercial representa una guerra implícita (FSW III 468) cuya extensión atenta contra la unidad interna y contra la autosuficiencia propias del Estado comercialmente cerrado; en efecto, su propuesta económica se orienta específicamente a lograr la plena autonomía: FSW III 482-485, 488, 500-501. 
reduce a la administración de lo dado, sino que transforma lo dado en función de las ideas, ha de mostrar el pasaje y cada uno de los puntos vinculantes entre el Estado real y el Estado racional, para hacer del último el concepto de fin del primero, para reconstruir el camino de la determinabilidad a la determinación.

La reconfiguración del Estado real a partir del Estado racional conduce a otro motivo central de la Doctrina de la Ciencia nova methodo: la primacía del querer o voluntad. En efecto, dice Fichte en esta obra: "El QUERER no puede ser deducido (...). Es algo originario, es todo lo más primario y lo más inmediato" (WLnm-H § 11, GA IV/2 113); y agrega: "en el QUERER nos manifestamos a nosotros mismos como produciendo algo nuevo (...). El querer aparece, según esto, como brotando desde una limitación autorrealizada de la facultad de la voluntad" (ídem, 114). En suma: "la voluntad es algo absoluto - [en ella] sujeto y objeto son uno y ciertamente lo mismo" (ídem, 115). ${ }^{11}$ Ante semejante poder productivo no hay realidad empírica que no sea transformable, y para ello sólo basta con disponer las limitaciones bajo la égida de la razón.

La destinación del hombre consiste precisamente en gestar este pasaje de lo determinable a lo determinado, en hacer real lo racional; en consonancia, concluye Fichte en la "Introducción" de El Estado comercial cerrado: "Todo lo bueno de lo que hombre debe ser partícipe, tiene que ser producido mediante su propio arte, a consecuencia de la ciencia; ésta es su destinación (Bestimmung)" (FSW III 398). ${ }^{12}$ Es su destinación tanto como su determinación, porque lo dado resulta de la actividad que lo produce. Así como el ámbito especulativo se articula con el técnico, del mismo modo el saber filosófico deviene arte de acción.

En este sentido, Fichte no sólo allana el camino del Estado real hacia el Estado racional, también dilucida la instancia que precede al Estado existente como retroproyección de un concepto determinado. En otras palabras, el Estado empíricoactual no es consecuencia de un designio trascendente, ni de fuerzas ocultas, etc., sino de la "anarquía comercial" que se opone radicalmente a "la institución del comercio conforme a la razón" (FSW III 476). La anarquía proviene del formalismo jurídico que, en nombre de la libertad, prescinde de la dimensión económica y de la organización social respectiva. Si el espacio relacional se sustrae de la articulación mediante leyes, entonces no sólo gana terreno un particularismo destructivo que tarde o temprano corroe el tejido social, sino también la hipóstasis de presuntas leyes inmutables que subyugan la voluntad y obligan a atenerse a los dictámenes del

11 La misma primacía del querer y de la voluntad caracteriza y atraviesa el Derecho natural: GNR Ein., GA I/3 326; § 1.Cor., GA I/3 332; § 7, GA I/3 387; § 8, GA I/3 400-401; § 16, GA I/3 435436. La coincidencia entre sujeto y objeto remite, en términos de Grundlage der gesammten Wissenschaftslehre (1794-5) (en adelante: GWL), al Yo absoluto: GWL § 1, GA I/2 259-260, 261 nota.

12 Desde luego, aunque las referencias se orientaron a la Doctrina de la Ciencia nova methodo, tomamos Bestimmung en el sentido de Bestimmung des Gelehrten (1794), GA I/3 28-31. 
mercado. Como dos caras de una misma moneda, el panorama no es otro que el del dogmatismo, y lo analizamos a continuación.

II.

El concepto de Estado racional que Fichte elucida en el primer libro de El Estado comercial cerrado se contrapone simultáneamente a dos sistemas falsos que anulan la voluntad colectiva e individual: el despotismo paternalista y el liberalismo cuasi-anárquico, ambos reticentes a la legislación racional. Esta doble contraposición se condice con la disputa frente al dogmatismo.

En la Primera introducción (1798) el dogmatismo es el sistema que parte de una cosa en sí como primer principio que fundamenta incluso al Yo y, por ende, lo vuelve heterónomo. Al tratarse del momento cumbre y soberano del cual se sigue todo lo demás, la cosa en sí determina la concatenación de los elementos en una secuencia mecanicista. ${ }^{13}$ Pero esta caracterización del dogmatismo, que bien se homologa a la crítica de Jacobi a Spinoza, contiene otra vertiente: para Fichte todos los filósofos modernos son dogmáticos (EE § 5, GA I/4 191 nota), salvo Kant o el kantismo (bien entendido). Y la disputa con los kantianos que dogmatizan el espíritu del kantismo reside en que, ante todo, sustentan un idealismo a medias, porque deducen las leyes del actuar a partir de la experiencia, como si detrás de los objetos hubiera una misteriosa cosa en sí que los justifique (EE § 7, GA I/4 202). Con ello el kantismo (mal entendido) proporciona otra versión del dogmatismo, en cuanto disemina la cosa en sí como fundamento de lo múltiple dado. La paradoja se trasluce en la Segunda introducción, donde Fichte argumenta que si el fundamento del sistema surge desde lo múltiple, entonces jamás se hallará la unidad del Yo:

¿De cuál Yo se habla aquí? Si [se habla] de algo que los kantianos juntan confiadamente desde la multiplicidad de representaciones, [algo] que no está en ninguna en particular, pero está en todas juntas (...); entonces Kant sería justamente un charlatán patético, como tales kantianos (ZE § 6, GA I/4 228). ${ }^{14}$

Los kantianos refieren directa o indirectamente a una suerte de organización espontánea de lo múltiple, que funcionaría con independencia de la unidad fundante y al modo de una mano invisible que todo lo ordena misteriosamente; de este esquema jamás se obtendrá un Yo, sino un engendro, un invento que restituye la cosa en sí. Al igual que el dogmatismo racionalista, el empirismo dogmatizado presupone algo extrínseco al Yo para fundar la unidad, en este caso: el ensamble de fragmentos. Ambas vertientes soslayan precisamente aquello que distingue al Yo: la autonomía,

13 Erste Einleitung in die Wissenschaftslehre (en adelante: EE), §§ 3-5, GA I/4 188-192.

14 Zweite Einleitung in die Wissenschaftslehre (en adelante: ZE). Un pasaje muy similar en WLnm-H § 12, GA IV/2 125-126. 
la autoposición, el para-sí, la facultad productiva. Para Fichte, en cambio, la unidad no se sigue ni se legitima desde la multiplicidad o lo dado, sino a la inversa: porque la unidad se pone a sí misma, hay un múltiple dado que se deduce de este ponerse. Es una articulación similar a la de lo proyectado y lo encontrado que analizamos en el apartado anterior. De la misma manera, la autoconciencia no condiciona la conciencia - es decir, no se reduce a la receptividad de contenidos extrínsecos que no la contradigan o anulen -, sino que la autoconciencia determina la conciencia, en el sentido de que todo el contenido está dado, fundado y producido, mediante las condiciones de la autoconciencia (ZE § 6, GA I/4 229). Por ende, si los kantianos pretenden aludir como fundamento de la experiencia algo que esté más allá del Yo, incurren en dogmatismo. Además, la unidad fundante no se circunscribe a administrar lo dado, porque el Yo no permanece ajeno a cada elemento múltiple, sino que está presente en cada elemento en función de la unidad.

En sintonía con esta doble contraposición, el despotismo paternalista equivale a la primera versión del dogmatismo, en cuanto la cosa en sí representa la determinación unilateral de lo social por parte de un poder soberano que se arroga exclusivamente la facultad de decidir la posición de los miembros, como si éstos fueran meras piezas de un mecanismo. A la vez, el soslayo de la unidad ante lo múltiple dado, la segunda versión del dogmatismo, equivale a someter al Estado al funcionamiento de una regularidad independiente y en apariencia extrínseca: el libre juego espontáneo de los particulares o las reglas del mercado. Pero el simple condicionamiento o administración de las reglas no alcanza a mostrar la verdadera función del Estado, que no se contenta con ordenar, sino que configura lo múltiple como un contenido suyo y lo determina.

En efecto, en El Estado comercial cerrado el lugar de la experiencia y de lo particular lo ocupa la economía, concebida como último eslabón de realización de lo filosófico-jurídico mediante lo político. No se trata de una instancia autónoma, sino del resultado de un proceso de determinación creciente (filosofía, derecho, política, economía), donde el espacio relacional-horizontal es consecuencia necesaria de la organización jurídico-política. El Estado racional, de acuerdo con los principios fundamentales del comercio supeditados al marco jurídico, se contrapone tanto al Estado despótico, unilateral y paternalista, que se arroga la facultad exclusiva de decidir sobre el bienestar y la felicidad de sus súbditos, como al Estado que sólo se encarga de proteger los derechos y posesiones individuales sin ninguna injerencia en el devenir relacional. Así, Fichte señala que el primero, el tutor de todos los asuntos individuales, fue suficientemente criticado, mientras que el segundo, que sólo se encarga de proteger lo establecido, además de estar excesivamente delimitado en sus potestades, contiene un equívoco importante, el de presuponer que la propiedad 
sea independiente del Estado. ${ }^{15}$ A partir de esta presuposición falsa se legitima la desigualdad que desencadena la anarquía.

Por una parte, el Estado despótico opera al modo de una cosa en sí o de un dogmatismo mecanicista que anula las voluntades que sólo pueden mantenerse sometidas a su ejercicio arbitrario, como en el fatalismo; por otra, el Estado liberal se condice con el idealismo a medias y con el empirismo de los kantianos que, sustrayendo lo múltiple de la unidad fundante, deben apelar a una regularidad misteriosa - la mano invisible del mercado - cuya detonante también es la cosa en sí, que siempre beneficia a los más poderosos. En el primer caso, la unidad ahoga y esclaviza la vitalidad de lo particular, mientras que en el segundo lo múltiple se legitima independientemente y sin unidad, como si cada uno de los agentes siguiendo su propio interés produjeran sin saberlo ni quererlo una armonía (el mercado) cuyo origen resulta inexplicable, salvo apelando a la cosa en sí. Contra estos dos polos y en consonancia con el idealismo que articula unidad y multiplicidad sin remisiones trascendentes, el espacio horizontal de lo particular se presenta para Fichte como ámbito de realización de la voluntad común mediante su dimensión institucionalizada, el Estado. De ahí que, como analizaremos en el punto siguiente, el espacio públicocomercial no sea un asunto de privados: ni del déspota sustraído de la igualdad, ni de los particulares reticentes a la unificación. Y como para Rousseau o Marx, para Fichte la clave reside en la propiedad:

En contraposición a esta opinión..." - según la cual el Estado debe desentenderse de las posesiones y del modo como han sido adquiridas por los particulares; esto es, la opinión según la cual la propiedad es independiente del Estado -, "yo diría: es la determinación del Estado primero dar a cada uno lo suyo, primero ponerlo en su propiedad, y sólo después también protegerlo (FSW III 399).

Si la legitimación de los derechos se efectúa independientemente del Estado y sólo en virtud de la relación sujeto-cosa, la escisión entre lo universal y lo particular se torna insuperable, y entonces toda unificación que no sea en beneficio del individuo se representa como obstaculizadora de su realización, entendida ésta como separada o contraria a los demás individuos. La propiedad anclada en el sujeto conlleva un Estado meramente nominal, que protege más a los que más poseen, y que conduce en última instancia a la anarquía y a la desintegración social, no sin agudizar al extremo la desigualdad. ${ }^{16}$ Por eso para Fichte la propiedad resulta significativa en el espacio relacional donde un conjunto de sujetos que comparten una misma esfera de libertad se delimitan sobre lo suyo y lo ajeno, es decir, la propiedad de cada uno se determina siempre en relación con todos los demás. Lo particular no surge como

15 FSW III 399. Esta misma secuencia reaparece en Die Grundzüge des gegenwärtigen Zeitalters (1805) (en adelante: GGZ), FSW VII 143-146, 150-152.

16 FSW III 441, 453, 508-509. Véase también: GNR § 21, GA I/4 91-92. 
un todo cerrado, fuera del cual están los otros, todos los cuales aspiran a expandirse y legitimar lo más que puedan (el estado de guerra); lo particular surge como un resultado, como parte de un todo, y como parte que sólo se puede comprender y explicar en el sistema de relaciones contenido en ese todo. Por tanto, el derecho concierne, no a la relación sujeto-cosa, sino a las relaciones entre personas, y éstas jamás se sustraen de la cuestión de la igualdad. ${ }^{17}$ En torno de la propiedad y de la igualdad el actor decisivo es el Estado; es, no el particular que se pone a sí mismo, sino la voluntad común que se sublima en el Estado la que pone a cada uno en posesión, porque el individuo jamás se realiza solo, sino únicamente mediante la relación con sus semejantes. El particular resulta mediado por lo universal. El individuo solo es nada, o es mera materia (GNR § 17.Cor., GA I/4 19-20). Por consiguiente, el derecho, que afecta al individuo y a sus condiciones vitales, llega a cada particular sólo mediante el tejido social. Tanto el derecho originario como el derecho de coacción del Fundamento del derecho natural se instituyen como ficciones heurísticas cuyo fundamento último, el Estado, contiene y neutraliza, porque delimita las esferas de libertad de los particulares y las articula en la universalidad de la voluntad común. Sin el Estado fundante, lo particular se revela incapaz de estabilidad, pues la lógica meramente horizontal se vuelve belicosa en el afán de asegurarse su posición frente al resto de los particulares sobre los que se extiende indefinidamente. ${ }^{18}$

El individuo no funda el Estado, no cede algo de sí para que el Estado lo reconozca y legitime su ventaja relativa frente a los demás; sino al revés: el individuo resulta puesto por el Estado en propiedad y de manera igualitaria, y antes de esta posesión legítima no tiene nada (GNR § 17, GA I/4 15 nota). Así, la recreación del momento fundacional implica necesariamente la puesta en propiedad de todos y cada uno en igual medida. Luego, la protección o poder coactivo, que en una sociedad desigual suelen evocar primero los que más poseen, en el Estado racional se revela secundario y, contrariamente a lo que podría suponerse, su evocación absoluta corresponde al pobre o al marginal. ${ }^{19}$ Pero lo importante reside en que el individuo no pone el Estado y, al mismo tiempo, en que éste no se enajena de aquél, porque el Estado es la unidad que contiene y explicita al individuo, que comprende lo social-económico como ámbito de realización de la voluntad común, y así lo protege también de las desavenencias materiales. Por tanto, el Estado no se convierte en lo trascendente que sólo capta el déspota o que se expresa en los decretos del mercado, ni surge

17 FSW III 402. En el ejemplo de Fichte, si en un territorio hay cien agricultores, la tierra deberá dividirse en cien parcelas iguales: FSW III 445. Para un estudio detallado del tema de la propiedad: D. James (2009) y (2011). Además, contra la pretensión de N. Nomer (2005, pp.54-55) de conciliar liberalismo y socialismo en Fichte, James concluye que la concepción fichteana de la propiedad se opone al liberalismo: (2009, pp.648-650, 660-665), (2011, pp.22-27).

18 FSW III 400. Fichte desarrolla los temas aludidos en GNR §§ 8-16.

19 "[E]l pobre" - dice Fichte - "tiene un absoluto derecho de coacción a la asistencia" (NR § 18, GA I/4 23). 
del mero vivir conjuntamente en un mismo espacio. El Estado configura la unidad fundante de relaciones organizadas:

Es el Estado, y únicamente él, el que reúne una multitud indeterminada de hombres en un todo cerrado, en una totalidad; es únicamente el Estado el que puede preguntarles a todos en su enlace [si pactan o no]; por tanto, únicamente mediante el Estado deviene primeramente fundada una propiedad legítima (FSW III 401). ${ }^{20}$

La totalidad manifiesta un enlace intrínseco, análogo al producto natural organizado, donde cada parte puede ser lo que es en virtud del todo que la vitaliza, y al mismo tiempo se vitaliza a sí misma contribuyendo a la totalidad a la que pertenece. En la medida en que el todo vive a través de sus partes, no las puede ni abandonar, ni aniquilar; no hay entre ellos contraposición ni lucha, sino sostenimiento y retroalimentación. $Y$ en la medida en que la parte vive gracias al todo, no se contrapone y disputa con otra parte su posición, sino que se contiene y armoniza en una actividad conjunta.

En consecuencia, la doble contraposición del idealismo - como unidad inmanente que articula en su interior lo universal y lo particular - frente al dogmatismo trascendente y al empirismo dogmatizado, permite comprender tanto el doble rechazo de Fichte al Estado despótico y al Estado liberal, como prevenir el riesgo de un Estado totalizante que anule la actividad de las partes y el de un Estado ausente y sometido a las reglas del mercado. En cuanto producción humana de voluntades unificadas en un mismo cuerpo social, el Estado no refiere ni a un designio dogmático, ni se subyuga a las leyes presuntamente independientes del comercio. Así, los dos ejes que tratamos hasta aquí (la idealidad fundante de realidad y la doble contraposición filosófica y política) confluyen en un tercero: la visión del espacio relacional-comercial como ámbito de realización de la voluntad común.

III.

La concepción fichteana del Estado racional incluye necesariamente a la economía, no como esfera de leyes autónomas e inmutables, ni como espacio exclusivo de los particulares, sino como ámbito de realización y aplicación de la voluntad común o del Estado. Dado que las leyes no se legitiman desde una regularidad incognoscible, y dado que la lógica relacional incide en la constitución y en la posición de cada particular, la economía forma parte directamente de la política, y el Estado no juega en ella el rol de un agente extrínseco, limitador, arbitrario, que distorsiona el presunto curso natural de las cosas (en tal caso, se reincide en

20 El Estado constituye el momento clave de la realización del derecho, porque el concepto de derecho concierne a las relaciones estables entre los individuos (GNR Ein., GA I/3 319; § 4.Cor., GA I/3 360; § 8, GA I/3 389), y el Estado se identifica bajo una dimensión institucional con ese entramado relacional (GNR § 16, GA I/3 435, 443-444). Lo mismo en GGZ, FSW VII 146-147. 
la escisión dogmática y despótica); el Estado es el agente central inmanente a esta misma dinámica vinculante.

De ahí que el contrato negativo que ordena a los particulares delimitarse y no inhibir la actividad del otro - actividad que, observada detenidamente, en cada parte se manifiesta conformando una misma actividad conjunta -, se completa y deviene contrato positivo en cuanto cada uno se compromete a colaborar con el otro, lo que en realidad también implica trabajar para sí mismo (FSW III 405). La clave de esta reversión contractual consiste en comprender que sí-mismo y otro son inescindibles, y que sólo se distinguen si se los capta en trabazón recíproca. Pero no se trata sólo de una trabazón formal, sino también de contenido, donde uno y otro son lo que son en la relación mutua, y entonces nadie puede beneficiarse (o perjudicarse) si a la vez no se benefician (o perjudican) también los otros, porque si alguien lo hace a costa de otro - es decir, contra el otro, separando su propia situación e interés -, o bien el Estado permite este nicho destructivo de lo común y del enlace, o bien funciona incluso como instrumento de opresión. Sin embargo, el Estado racional, en cuanto garante de lo común y de la igualdad, existe precisamente para que un particular no se sobreponga al resto y subyugue la lógica relacional a su propio interés.

Ahora bien, en el despliegue de este tercer punto de nuestro análisis (el espacio público comercial), volver a contraponer el Estado y los intereses particulares, o insistir en que la política no debe inmiscuirse en el comercio, o que Estado e igualdad están en carriles diferentes, etc., significa desconocer absolutamente la ilación del pensamiento de Fichte. Ni se trata de un actor que coacciona al particular a actuar de determinada manera, ni de una política que se entromete en la economía, ni de un igualitarismo coactivo-compulsivo. En efecto, el segundo capítulo del primer libro de El Estado comercial cerrado se titula: “Aplicación universal de los principios fundamentales establecidos sobre el intercambio público" (FSW III 403); por lo tanto, conceptualmente ninguno de los estamentos (productores, artesanos, comerciantes, etc.) puede sentirse compelido a entregar los productos, salvo que escindan el beneficio propio y lo sobrepongan a la situación de los demás, gestando así el ventajismo y la opresión. Este razonamiento egoísta confunde la parte (el individuo o el estamento) con la totalidad, e invierte la relación: pone la totalidad al servicio de la parte. Por el contrario, en el Estado racional fichteano prima la colaboración recíproca donde la situación de cada parte está sujeta a la situación de todas las otras partes. Así, el Estado evita los dos polos que distorsionan la horizontalidad cooperativa: el lujo y el empobrecimiento. ${ }^{21}$ Porque uno y otro son inescindibles: es la misma colaboración recíproca - en un Estado de igualdad formal y que se desentiende de la cuestión económica - la que conlleva el enriquecimiento de algunos y el consiguiente

21 FSW III 408-409. Desde luego, la temática incluye los precios fijos (FSW III 415-416) y el control del valor de cambio, que en principio sería el grano y después el dinero (FSW III 435). 
empobrecimiento de otros. Y la variable que podría soslayar la organización interna, el comercio exterior, también cae bajo la racionalidad estatal, lo que no significa insistimos - anular el intercambio, sino someterlo a la voluntad común y no dejarlo a la conveniencia de unos pocos. ${ }^{22}$ En esta perspectiva, el Estado se caracteriza por ser el factor clave de la estabilidad del sistema de relaciones que envuelven a los particulares, el actor decisivo del sostenimiento de la reciprocidad.

Los intérpretes del pensamiento político de Fichte suelen oscilar entre una visión más armonicista, centrada en la primera parte del Fundamento del derecho natural, y una visión más belicista y hobbesiana de la intersubjetividad, sustentada en la aplicación del concepto de derecho. ${ }^{23}$ Mientras la primera soslaya el drama político (el conflicto, la tensión, la articulación) y la relevancia del Estado, la segunda dificulta la sociabilidad y promueve un concepto de Estado centrado en la coacción. Más allá de que las dos perspectivas no se excluyen y se neutralizan mutuamente, de una o de otra manera se necesita una instancia de unificación universal, en el primer caso porque la armonía no exime la posibilidad de opresión, y en el segundo porque la guerra de todos contra todos destruye incluso la individualidad. La instancia de unificación, la voluntad común que se concretiza a través del Estado, significa la puesta en unidad de las voluntades particulares, que ya no han de concebirse de modo escindido e independiente, sino como elementos de una misma totalidad. Pero esta unificación no aniquila la particularidad, ni la deja librada a una autoregulación espontánea, precisamente porque no anula totalmente el conflicto, ni se desentiende de él.

La articulación entre los elementos contrapuestos y la unidad que los contiene corresponde no sólo a las consideraciones filosófico-jurídicas de Fichte, sino también al corazón de su metafísica. En efecto, en el tercer principio del Fundamento de toda la Doctrina de la Ciencia (1794) Fichte se propone conjugar dialécticamente el Yo y el No-Yo, para lo cual éstos han de ceder su carácter inicial de absolutos o plenitudes excluyentes y limitarse o dividirse en cierta medida. En la anulación parcial Yo y No-Yo, ahora determinados, se contraponen entre sí y a la vez en referencia al Yo absoluto; es decir, el Yo y el No-Yo finitos se entablan en reciprocidad. Este proceso de contraposición recíproca no se suscita por fuera del Yo, sino dentro de él y como

22 FSW III 420. En esta misma línea, C. Dierksmeier (2011, pp.114-122) argumenta que la intersubjetividad no se sostiene sin la institución del Estado, que es el que genera estabilidad y unifica los diversos fines ( $y$, en tal sentido, no se contrapone, ni interfiere, ni obstaculiza los intereses particulares), y que por eso Fichte jamás quiere renunciar al Estado de Bienestar y a la justicia. Sin embargo, sostiene que se trata de un modelo estático de racionalidad económica, que Fichte recién tornará dinámico en la Rechtslehre de 1812, donde el comercio forma parte directamente de la función pública.

23 Esta contraposición entre las dos primeras secciones del Derecho natural (GNR §§ 1-7) y la tercera (GNR §§ 8-16) proviene de L. Siep (1979, p.35), (1992, pp.42-43 ss.), que trata de distanciarse y matizar el hobbesianismo de la interpretación de R. Schottky (1995, p.176). R. Williams (1992, p.64) sigue la perspectiva de Siep (1979) y propone una mediación intersubjetiva y ética. 
un alumbramiento y especificación interna de sus determinaciones (GWL § 3, GA I/2 268-272). En el círculo sistemático de la Doctrina de la Ciencia (la filosofía) todo elemento queda enlazado y distinguido en base al principio de fundamento que se sigue del tercer principio y que establece que los opuestos se identifican en un aspecto (se relacionan) y que los idénticos se oponen en un aspecto (se distinguen), lo que posibilita un procedimiento sintético de buscar las notas comunes a los elementos y un procedimiento antitético o analítico centrado en las contraposiciones (GWL $\S 3$, GA I/2 272-274, 276).

En la posterior Doctrina de la Ciencia nova methodo, Fichte reelabora la exposición del Fundamento colocando el tercer principio en correlación con el segundo, porque negación y determinación son lo mismo: "La determinación del Yo, de la que se parte, es al mismo tiempo también el medio de vinculación entre Yo y No-Yo" (WLnm-H § 2, GA IV/2 41). Para que el No-Yo pueda ser puesto, el Yo tiene que ser co-puesto (mitgesezt), y así los elementos contrapuestos que desde un punto de vista horizontal parecen extrínsecos, desde el punto de vista vertical y unificador están en el Yo. La multiplicidad existe y convive en lo Uno. Por ende, la limitación o divisibilidad significa que en el Yo la multiplicidad está separada y reunida, sin que ello implique la diversificación o ruptura de la unidad fundamental. Y las partes son partes no sólo porque se contraponen mutuamente (tanto porque armonizan como porque entablan conflictos entre sí), sino también porque están contenidas en una misma unidad (WLnm-H \& 2, GA IV/2 42-43).

Según esta exposición, lo determinado se deduce de lo determinable, y lo mismo ocurre con la intersubjetividad. El punto más alto al que llega la Doctrina de la Ciencia nova methodo, la comunidad o reino de seres racionales, se erige como ámbito referencial del ser racional finito: sólo a partir de una dimensión inteligible compartida el individuo puede encontrarse como tal, esto es, pensando a otros y siendo pensado por otros, sus iguales (WLnm-H § 12, GA IV/2 141-143). Aquí se juega la simultánea distinción y relación entre partes. Lo determinado, el individuo, se deduce de lo determinable, el reino racional, y se reconoce a sí mismo en el acto de separarse y equipararse con los otros; sólo aquí comienza la autoconciencia. Por consiguiente, la razón (lo inteligible) se manifiesta en el individuo (sensible), y éste no se puede explicar de modo aislado: "ningún ser racional particular puede existir para sí mismo, [sino que] existe sólo como parte mediante y para el todo" (WLnm-H $\S 15, \mathrm{GA}$ IV/2 177). De ahí que la mismidad (Selbstheit) se active desde la referencia a la razón y desde la exhortación (Aufforderung) del otro. La reciprocidad involucra una articulación horizontal-vertical. Concluye Fichte: "el INDIviduo se puede desarrollar a sí mismo sólo mediante otro [individuo] desarrollado y el desarrollo de los primeros INDIVIDuos se puede explicar sólo mediante el supuesto de una razón absoluta suprema" (ídem, 178). 
En términos de la Doctrina de la Ciencia, el individuo que utiliza al otro y se beneficia del perjuicio de otro, somete la reciprocidad a su propio interés egoísta, se apropia de la Yoidad o razón como de una prerrogativa exclusiva, y deviene déspota y opresor. Se trata del individuo que confunde su yo con el Yo y del que Fichte nos previene tanto en el comienzo del Fundamento del derecho natural como en la Segunda introducción. ${ }^{24}$ Por ende, para esta concepción resulta tan importante elevarse a la Yoidad (la universalidad inmanente) como reconocerse formando parte de una articulación recíproca horizontal (saberse particular). Sólo conjugando los dos aspectos se estabilizan y unifican las múltiples actividades en un mismo Estado. $Y$ éste no remite a una entidad trascendente, sino que es el totum concebido en términos de actividad. El ánimo colaborativo de sujetos activos que se potencian mutuamente al reconocerse como elementos de una sola actividad-total que los contiene y posibilita, constituye un rasgo crucial del pensamiento de Fichte, y se encuentra también aquí, en El Estado comercial cerrado:

En este Estado todos son servidores del todo, y reciben por esto su parte justa en los bienes del todo. Ninguno se puede enriquecer singularmente, pero tampoco puede empobrecerse. A todos los individuos se les garantiza la continuidad de sus situaciones, y de este modo se garantiza la tranquila e igualitaria continuidad del todo (FSW III 419). ${ }^{25}$

A diferencia del Estado liberal que se desentiende de las posiciones de los particulares y permite y/o colabora en las presuntas contingencias de los mandatos del mercado, el Estado racional se caracteriza por asumir la reciprocidad como ámbito de realización de lo común y armonizar los intereses contrapuestos en función de la unidad igualitaria y estable de todos los elementos. Por lo tanto, el espacio público comercial no se presenta como un vacío a llenar por el libre juego espontáneo de sujetos que sólo persiguen la expansión de sus posesiones, sino como el flujo común que afecta y atraviesa el sostenimiento de cada particular y que requiere necesariamente una visión de conjunto para poner y mantener el articulado de coincidencias y discrepancias. Para Fichte no se trata sólo de una cuestión política (cómo lograr una consistencia duradera), sino también filosófica y metafísica, donde lo relacional prima sobre lo particular, donde el individuo sólo puede ser deducido de un entramado vinculante, y donde la lógica egoísta representa una subversión ficcional y destructiva que, llevada al extremo y pensada en su precisa medida, se consume incluso a sí misma. De todos modos, así como la razón no aniquila al ser racional finito, sino que lo deduce y explica, la unificación estatal no extirpa el conflicto, simplemente lo neutraliza, lo deduce al interior del totum, y lo resuelve

24 GNR Ein., GA I/3 313. ZE §§ 8-9, GA I/4 253-256.

25 Un pasaje muy parecido en Bestimmung des Gelehrten (GA I/3 49) y en Die Bestimmung des Menschen (1800): FSW II 277. Véase también GGZ, FSW VII 151, 160-161. 
de manera política.

En suma, la dimensión colaborativa de actividades múltiples entrelazadas en una misma totalidad social-estatal expresa la aplicación de la reciprocidad en el marco de la unidad fundante, unidad que no se legitima desde una cosa en sí trascendente ni desde la regularidad incognoscible, sino como articulación inmanente de los elementos contenidos en ella, donde lo empírico y determinado resulta de un proceso que parte de la determinabilidad y se concretiza a través de la transformación de las limitaciones. Los tres aspectos analizados (la idealidad como fundamento de la realidad, la doble contraposición ante el dogmatismo en sintonía con la perspectiva de la Doctrina de la Ciencia, y el espacio público comercial como instancia de realización de la política) muestran que la concepción del Estado racional de Fichte se sustenta en su visión metafísica y filosófica.

\section{Referencias}

1) Fuentes, obras y abreviaturas:

1.a) Obras completas:

Fichte, J. (1962ss.). Gesamtausgabe der Bayerischen Akademie der Wissenschaften. Hrsg. R. Lauth y otros. Stuttgart: Fromman. (GA, serie/tomo, página). (1971). Sämmtliche Werke. Hrsg. I. Fichte. Segunda edición. Berlín: W. de Gruyter. (FSW, tomo, página).

1.b) Obras de Fichte:

. Zurückforderung der Denkfreiheit (1793), en FSW VI 1-35.

- Beitrag zur Berichtigung der Urteile des Publicums über die französische Revolution (1793), en FSW VI 37-288.

$23-74$ . Einige Vorlesungen über Bestimmung des Gelehrten (1794), en GA I/3 249-451. (GWL). - Grundlage der gesammten Wissenschaftslehre (1794-5), en GA I/2 . Ueber Geist und Buchstab in der Philosophie (1795), en FSW VIII 270300. 266. (WLnm-H).

$$
\text { Wissenschaftslehre nova methodo - Halle (1796 ss.), en GA IV/2 17- }
$$
. Grundlage des Naturrechts (1796-7), en GA I/3 313-469, I/4 5-165. (GNR). . Erste Einleitung in dieWissenschaftslehre (1798), en GA I/4 183-208. (EE). 269. (ZE). Zweite Einleitung in dieWissenschaftslehre (1798), en GA I/4 209- 
Der geschlossene Handelsstaat (1800), en FSW III 387-513.

. Die Bestimmung des Menschen (1800), en FSW II 165-319.

1-256. (GGZ).

. Die Grundzüge des gegenwärtigen Zeitalters (1805), en FSW VII

2) Bibliografía secundaria.

Dierksmeier, C. (2011). Der Staat und die Wirtschaft. Fichtes politische Ökonomik. En: G. Zöller (Hrsg.). Der Staat als Mittel zum Zweck. Fichte über Freiheit, Recht und Gesetz. Baden-Baden: Nomos.

Frischmann, B. (2006). Die Herausbildung des Sozialstaatsdenkens im neuzeitlichen Kontraktualismus von Hobbes bis Fichte. Zeitschrift für philosophische Forschung, 60(4), pp.554-589.

Gray, R. (2003). Economic Romanticism: Monetary Nationalism in J. G. Fichte and A. Müller. Eighteenth-Century Studies, 36(4), pp.535-557.

Gross, S. (2008). Handelsstaat versus Globalisierung. Anmerkungen zu J. G. Fichtes «Geschlossnem Handelsstaat». Información Filosófica, 11, pp.201-215.

James, D. (2009). Applying the concept of right: Fichte and Babeuf. History of political thought, $X X X(4)$, pp.647-677. University Press.

Moggach, D. (2009). Freedom and Perfection: The German Debates on the State in the Eighteenth Century. Canadian Journal of Political Science, 42(4), pp.10031023.

Nakhimovsky, I. (2011). The Closed Commercial State. New Jersey: Princeton University Press.

Nomer, N. (2005). Fichte and the Idea of Liberal Socialism. The Journal of Political Philosophy, 13(1), pp.53-73.

Schottky, R. (1995). Untersuchungen zur Geschichte der staatsphilosophischen Vertragstheorie im 17. und 18. Jahrhundert (Hobbes, Locke, Rousseau und Fichte). Segunda edición. Ámsterdam / Atlanta: Rodopi.

Siep, L. (1979). Anerkennung als Prinzip der praktischen Philosophie. Freiburg / München: Karl Alber.

Suhrkamp.

(1992). Praktische Philosophie im Deutschen Idealismus. Frankfurt:

Verzar, A. (1980). Das autonome Subjekt und der Vernunftstaat. Bonn: Bouvier.

Williams, R. (1992). Recognition. Fichte and Hegel on the Others. New York: State University of New York Press.

Willms, B. (1967). Die totale Freiheit. Köln / Opladen: Westdeutscher Verlag. 